\title{
Electric Vehicle Routing Problem in Urban Logistics
}

\author{
Yiwei Lu*
}

Shanghai University, Shanghai 200444, China. E-mail: luyiwei1997@163.com

\begin{abstract}
Due to the impact of global warming, diesel locomotives that use fossil energy as fuel are gradually being replaced by electric vehicles. At present, many countries at home and abroad are actively promoting the development of the electric vehicle industry in response to the call of the Paris Agreement. However, electric vehicles have a maximum mileage limit, so the reasonable layout of electric vehicle charging stations is also a problem to be solved today. In this article, the author analyzes the research background of the electric vehicle routing problem. After introducing several new research directions in the current electric vehicle routing problem, we propose an optimization algorithm for solving those types of problem. It brings certain theoretical significance for future generations to solve the problem of electric vehicle routing in real life.
\end{abstract}

Keywords: Vehicle Routing Problem; Urban Logistic; Charging Station

\section{The background of electric vehi- cle routing problem}

In recent years, the progress of social productivity, the rapid development of the Internet and the increasing living standards of the people have continuously promoted the vigorous development of e-commerce. More and more consumers are more obsessed with shopping directly on online shopping platforms such as Taobao and JD, instead of shopping in offline physical stores. Under such a development background, especially during the prevention and control of the COVID-19, the whole country has responded to the call of the party and the state to independently retreat and stay at home. However, the process of home isolation also requires supplies of living materials, so consumers' active home isolation directly gives birth to a kind of "home econo- my" - online shopping. Online shopping has also become an important way for households to maintain their daily production and life.

Although the rapid development of China's Internet has driven the development of China's express service industry, there are also many urgent problems in the express service industry at this stage, which also puts forward higher requirements for the level of logistics services. First of all, in terms of customer satisfaction, consumers who shop online often complain about the inconsistency of delivery services and the inconsistent delivery of packages purchased online. The last mile of delivery has many characteristics such as large express orders volume, repeated distribution routes, small order batches, and scattered customer locations. In this case, the vehicle has to deliver packages from the distribution center to

Copyright (C) 2020 Yiwei Lu

doi: $10.18686 /$ utc.v6i4.96

This is an open-access article distributed under the terms of the Creative Commons Attribution Non-Commercial License

(http://creativecommons.org/licenses/by-nc/4.0/), which permits unrestricted non-commercial use, distribution, and reproduction in any medium, provided the original work is properly cited. 
multiple customer nodes multiple times a day. For the customer, the time for the customer to receive the package is limited by a time window, that is, a customer can only receive goods within the specified time window. Therefore, the above conditions will directly affect the time for customers to receive goods. The last mile of delivery is very important to improve consumers' online shopping experience. Secondly, in terms of energy saving and emission reduction, it is well known that ordinary express delivery vehicles now use internal combustion engines to burn fossil energy to brake. Fossil energy is a non-renewable, non-clean energy, and it is not an inexhaustible energy source. At the same time, inadequate combustion of fossil energy will emit a large amount of harmful gases or harmful substances including carbon monoxide, nitrogen oxides and particulate matter, which will damage the air quality of cities, exacerbate the "greenhouse effect" and endanger people's health. At present, our country is actively promoting the promotion of new energy vehicles. Many companies have replaced their existing internal combustion engine logistics vehicles with electric vehicles. Compared with internal combustion engine logistics and distribution vehicles, electric logistics and distribution vehicles are cleaner and more environmentally friendly. They only need to be fully charged before they can continue to participate in logistics and distribution tasks. With its advantages of stable operation, low noise, and almost zero emission, electric vehicles can meet the requirements of vehicles in any road environment and become the mainstream of future transportation. Because electric vehicles have battery capacity constraints, this further limits the total journey of electric vehicles. In the current urban planning system, the number of charging stations is significantly less than the number of gas stations, and the layout of the charging stations in the city is messy, making it impossible to charge electric vehicles in time when there is a lack of electricity. Since electric vehicles need to be charged, the charging time is significantly longer than the refueling time of internal combustion engine vehicles, which will result in goods not being delivered to customers on time. Therefore, the occurrence of these situations will cause the goods to be unable to be delivered to customers in an efficient way. Then, the electric vehicle routing problem in urban logistics distribution is proposed.

\section{The new research direction of electric vehicle routing problem}

\subsection{Electric vehicle routing problem based on dynamic logistics information}

At present, most of the electric vehicle routing problems are a statically determined system. However, in actual logistics and distribution, the statically determined system has many drawbacks in the processing of various information. Common routing problems need to deal with dynamic changes such as changes in customer demand for orders, changes in customer information, changes in demand for delivery goods, delivery time, traffic conditions, and charging station status. The statically determined system cannot effectively process this kind of dynamic information in a timely manner. Therefore, the related research on how to deal with dynamic uncertain systems is still in a relatively blank area, which is a new research direction for the field of electric vehicle path optimization problems. In the current research, the problem input required in the solution method, as well as the optimization of information processing and optimization constraints are in a relatively mature stage. The difficulty here is how to integrate dynamic information into the solution method based on the premise that the vehicle is an electric vehicle.

\subsection{Electric vehicle routing problem consid- ering charging restrictions}

In terms of the characteristics of charging piles for electric vehicles, the current charging equipment and charging technology of electric vehicles do have a bottleneck compared with traditional energy vehicles. When electric vehicles are charged in areas with congested roads and heavy traffic, the electric vehicles need to wait a long time at the charging station because there are not enough charging piles. In the face of this difficulty in charging, a more mature solution is to centrally feed back the information of electric vehicles and charging stations that have charging needs to the terminal, and the terminal will add the information to the vehicle path optimization system, which will satisfy the charging of electric vehicles. Demand is an important part of path optimization.

In addition, the overall safety impact of the charging process of the charging station on the grid needs to be 
scientifically evaluated. Generally, when electric vehicles are charged, the power grid needs to bear a large amount of electricity consumption. Therefore, when a large number of electric vehicles are in charging state, the pressure on the power grid will increase significantly, while resulting in power safety risks. If electric vehicles of a certain scale are established in the logistics system, the emergence of electric vehicles will not only exacerbate traffic congestion, but also pose new challenges to the power system.

The two kinds of pressures caused by the charging of electric vehicles in the transportation network and the power grid at the same time are a new derived problem. This situation has not been regarded as a major topic by the research of the electric vehicle logistics industry. The reality is that when establishing a charging station, how to take into account both the transportation network and the power network and select the location of the charging station is still the primary issue in the development of the industry. How to optimize the balanced relationship between the transportation network, the power grid and the charging pile is an urgent problem to be solved. The establishment of fast charging stations should take into account the cost of charging stations and the difficulty of upgrading the power grid. Therefore, the solution of electric vehicle routing problem and the solution of charging station location problems should be comprehensively considered.

\subsection{Electric vehicle routing problem for electric vehicles in different application fields}

In the current practical application of electric vehicles, the public transportation system is the most widely used for electric vehicles, and it is also the key area of electric vehicle research today. At present, the share of electric vehicles among public buses in large and medium-sized cities in China is rising sharply, while common electric buses can only be charged at designated stations. More advanced electric bus charging systems use charging sensing devices for instant charging when passengers get on and off the bus. However, to fully realize this technology charging, many bus station charging systems are required. There are a lot of bus stations in big cities. If all bus station charging stations are built, the cost is too high. Therefore, the location of electric bus charging stations is a new research problem.

Compared with urban charging, in long-distance transportation, such as highways, the charging of electric vehicles faces more problems. In order to solve these problems, it is necessary to share vehicle charging information and charging station information to solve this vehicle problem through data analysis.

At present, a new form of shared transportation service is gradually taking shape. This is a new type of service that can change many industries in the future. All cars in the city may become vehicles in the future. Therefore, studying the dynamic shared vehicles routing problem is also an important research topic at the moment.

\section{The solution method of electric vehicle routing problem}

The mathematical model of the electric vehicle routing problem is a mixed integer programming model, so solving electric vehicle routing problem is essentially to solve a mixed integer programming model. At present, there are many methods to solve mixed integer programming problems. The situation of the solutions obtained by these methods can be divided into two categories: precise algorithms and heuristic algorithms. These algorithms are specifically used to solve the vehicle routing problem as follows.

\subsection{Precise algorithm}

\subsubsection{Branch and bound method}

The branch and bound method was proposed by Land and Doig in the $1960 \mathrm{~s}^{[1]}$, while was specially used to solve a class of pure integer programming problems or mixed integer programming problems. The main solution steps of the branch and bound method are as follows: first, solve the corresponding relaxed linear programming problem LP of the integer linear programming problem ILP, and randomly select a component $x$ that does not meet the integer condition in the optimal solution of the solved relaxed linear programming problem LP. Assuming that the value of the component $x$ is $L$, [L] represents the largest integer not exceeding L, where [] is the sign of rounding down. Now construct two linear programming constraints by rounding conditions: $x \leqslant$ $[\mathrm{L}]$ and $\mathrm{x} \geqslant[\mathrm{L}]+1$. Then these two new constraints are added to the previously solved relaxed linear program- 
ming problem LP, then the LP problem is divided into two sub-problems LP1 and LP2. Then the same as the previous steps, continue to ignore the integer constraints of LP1 and LP2, and solve the relaxed integer linear programming problems LP1 and LP2 respectively. According to whether the subsequent solution meets the integer condition, the branch is constructed according to the previous method, and so on. When all the components of the final solution are integers, then it is the optimal solution of the integer programming.

To sum up, the branch and bound method mainly starts from solving the corresponding relaxed linear programming problem, and first solves its optimal solution. If there is a component that does not meet the integer condition in the obtained optimal solution, one of the components is decomposed into two constraints and added to the original problem respectively to form two linear programming problems, and the feasible region of understanding is reduced at this time. Then continue to solve the two new linear programming problems until all the components of the optimal solution are integer solutions.

\subsubsection{Cutting plane method}

The cutting plane method was proposed by the American scholar R. E. GoMory (1958) and is suitable for solving integer programming problems. The cutting plane method is similar to the branch and bound method, and it is the optimal solution to solve the relaxation problem first without considering the integer constraints of the integer programming problem model. If the optimal solution obtained by solving is an integer solution, the solution process stops, and the solution is the optimal solution of integer programming. If the optimal solution obtained by the solution contains a non-integer part, then a new constraint condition must be added to the non-integer solution part before continuing the solution. This new constraint is the cutting equation. The function of the cutting equation is to cut the feasible region of the partial non-integer solution of the relaxation problem, and then retain all the integer parts of the problem. After several times of cutting the feasible region, the entire feasible region may only have all components as integer vertices, and this vertex is the integer optimal solution of the integer programming problem.

\subsection{Heuristic algorithm}

\subsubsection{Genetic algorithm}

The genetic algorithm first produces a set of initial solutions called the first species population, and then according to the principle of survival of the fittest in nature, it successively generates and selects the solution suitable for the population as the feasible solution of the problem $^{[2]}$. In each iteration of the search process, individuals in the population generate new individuals through genetic crossover and mutation, and then retain better individuals according to the size of individual fitness, thereby gradually forming a new population. This process will cause the population to gradually produce individuals adapted to the environment like the evolutionary process in nature, until the population meets the defined convergence conditions and terminates, thereby obtaining a satisfactory solution to the problem.

\subsubsection{Tabu search algorithm}

Tabu search algorithm has the advantages of avoiding repeated searches and solving the problem quickly because of the characteristics of memory solution ${ }^{[3]}$. In order to avoid roundabout searches, a storage structure Tabu table is introduced in the algorithm to store the better solutions that have appeared during the search process. In the subsequent search process, the algorithm will avoid the neighbors that have searched for better solutions. Domain, this avoids the repeated search of the algorithm to a certain extent. At the same time, in order to prevent the Tabu table from shielding some good solutions, the algorithm sets amnesty criteria to pardon some good solutions.

The Tabu search algorithm can memorize better solutions while ensuring search diversity, so as to achieve the goal of global optimization after neighborhood operations.

\subsubsection{Simulated annealing algorithm}

In order to solve the local optimal solution problem, Kirkpatrick proposed the simulated annealing algorithm which can effectively solve the local optimal solution problem $^{[4]}$. In molecules and atoms, the more energy is greater, the more molecules and atoms are unstable, and the more energy is lower, the more atoms are stable. Annealing is a physics term that refers to the process of heating and cooling an object. The simulated annealing algorithm comes from the process of crystal cooling. If the solid is not in the lowest energy state, heating and then cooling the solid, as the temperature slowly de- 
creases, the atoms in the solid are arranged in a certain shape to form high-density, low-energy regular crystals, corresponding to the global optimal solution in the algorithm. And if the temperature drops too fast, it may cause the atoms to lack enough time to arrange into a crystalline structure, resulting in an amorphous with higher energy, which is the local optimal solution. Therefore, according to the annealing process, add a little energy to it, and then cool it. If the energy is increased and the local optimal solution is jumped out, then this annealing is successful.

\section{Conclusion}

The acceleration of Chinese urbanization process and the rapid development of e-commerce have promoted the development of urban logistics and distribution. However, the shortcomings of urban logistics distribution are increasingly apparent, especially in the logistics distribution process, the impact of the distribution process on transportation and the environment is particularly obvious. With its advantages of low energy consumption and low pollution, electric vehicles play a positive role in solving the adverse effects of urban distribution on cities. However, compared with traditional diesel locomotives, electric vehicles have the constraint of total mileage, and there is a greater risk of insufficient power during the distribution process, which will lead to a small distribution service range and low quality of electric vehicles. Based on these considerations, this article proposes some new electric vehicle routing problems in actual logistics and distribution, and summarizes some existing methods to solve the vehicle routing problem for future research and use.

\section{References}

1. Land AH, Doig AG. An automatic method of solving discrete programming problems. Econometrica 1960; 28(3): 497-520.

2. Holland JH. Adaptation in natural and articial systems. U.S.: University of Michigan Press; 1975.

3. Glover F. Tabu search - Part I. ORSA Journal on Computing 1989; 1(3): 135-206.

4. Kirkpatrick S, Gelatt Jr. CD, Vecchi MP. Optimization by simulated annealing. Science 1983; 220(4598): 671-680. 